\title{
Reconstructing neural representations of tactile space
}

\author{
Luigi Tamè a,b,*, Raffaele Tucciarelli ${ }^{\text {a }}$, Renata Sadibolova ${ }^{\mathrm{a}, \mathrm{c}}$, Martin I. Sereno ${ }^{\mathrm{a}, \mathrm{d}, \mathrm{e}}$, \\ Matthew R. Longo ${ }^{\mathrm{a}, * *}$ \\ ${ }^{a}$ Department of Psychological Sciences, Birkbeck, University of London, London WC1E 7HX, UK \\ ${ }^{\mathrm{b}}$ School of Psychology, University of Kent, Canterbury CT2 7NP, UK \\ ${ }^{c}$ Department of Psychology, Goldsmith, University of London, London, UK \\ ${ }^{\mathrm{d}}$ University College London, University of London, London, UK \\ ${ }^{\mathrm{e}}$ San Diego State University, San Diego, USA
}

\section{A R T I C L E I N F O}

\section{Keywords:}

Shape of the skin

Tactile distance perception

Somatosensory and motor cortices

Multidimensional scaling

Body distortions

fMRI

Multivariate analysis

\begin{abstract}
A B S T R A C T
Psychophysical experiments have demonstrated large and highly systematic perceptual distortions of tactile space. Such a space can be referred to our experience of the spatial organisation of objects, at representational level, through touch, in analogy with the familiar concept of visual space. We investigated the neural basis of tactile space by analysing activity patterns induced by tactile stimulation of nine points on a $3 \times 3$ square grid on the hand dorsum using functional magnetic resonance imaging. We used a searchlight approach within pre-defined regions of interests to compute the pairwise Euclidean distances between the activity patterns elicited by tactile stimulation. Then, we used multidimensional scaling to reconstruct tactile space at the neural level and compare it with skin space at the perceptual level. Our reconstructions of the shape of skin space in contralateral primary somatosensory and motor cortices reveal that it is distorted in a way that matches the perceptual shape of skin space. This suggests that early sensorimotor areas critically contribute to the distorted internal representation of tactile space on the hand dorsum.
\end{abstract}

\section{Significant Statement}

Here, we show that the primary somatosensory and motor cortices, rather than higher-level brain areas, are critical to estimating distances between tactile stimuli on the hand dorsum. By combining functional magnetic resonance, Procrustes alignment, and multidimensional scaling, we reconstructed the shape of skin space in the brain. Strikingly, the shape of the skin that we reconstructed from neural data matches the distortions we found at the behavioural level, providing strong evidence that early sensorimotor areas are critical for the construction of tactile space. Our work therefore supports the view that tactile distance perception is computed at earlier stages in the somatosensory system than is usually supposed.

\section{Introduction}

Perceiving the physical properties of objects through touch is critical for everyday behaviour. By measuring tactile perception, we learn not only about perceived properties of external objects but also about the organisation of the somatosensory system. In particular, the perception of tactile distance has been widely used to investigate the somatosensory system. One source of information about the mechanisms underlying tactile distance perception comes from studies of tactile distance illusions indicating that the representation of the skin surface is systematically distorted. For example, in his seminal work Weber (1834/1996) found that when moving the two points of a compass across the skin, the perceived distance changed, feeling larger on more sensitive skin regions (e.g., the hand) than on less sensitive regions (e.g., the forearm). This effect is known as Weber's Illusion, and subsequent studies have found a systematic relation across the skin between cortical magnification factors and perceptive tactile distance (Cholewiak 1999; Taylor-Clarke et al., 2004). This suggests that distortions of primary somatotopic maps, for example of the famous Penfield homunculus (Penfield and Boldrey 1937), are preserved in some aspects of higherlevel tactile perception. Therefore, a complete process of tactile size constancy may not always be achieved.

Analogous distortions are also found when a single skin region is stimulated in different orientations. For instance, Longo and Haggard (2011) found a bias to overestimate the distance between touches

\footnotetext{
* Corresponding author at: School of Psychology, University of Kent, Canterbury CT2 7NP, UK.

** Corresponding author at: Department of Psychological Sciences, Birkbeck, University of London, London WC1E 7HX, UK. E-mail addresses: 1.tame@kent.ac.uk (L. Tamè), m.longo@bbk.ac.uk (M.R. Longo).
} 
oriented with the medio-lateral hand axis compared to the proximodistal axis. Similar anisotropies have been reported on several other body parts, including the forearm (Green 1982; Marks et al., 1982; Knight et al., 2014), thigh (Green 1982; Tosi and Romano 2020), shin (Stone et al., 2018), and face (Longo et al., 2015a; Fiori and Longo 2018; Longo et al., 2020). Intriguingly, such illusions mirror anisotropies in the geometry of tactile RFs which tend to be oval-shaped both in the spinal cord (e.g., Brown et al., 1975) and in SI (e.g., Alloway et al., 1989; Brooks et al., 1961) with the longer axis aligned with the proximo-distal body axis.

Recent results have shown that tactile distance is susceptible to sensory adaptation (Calzolari et al., 2017), suggesting that it might be a basic feature coded at relatively early stages of somatosensory processing. Indeed, there is evidence that perceived tactile distance is shaped by low-level features of somatosensory organization such as cortical magnification (Cholewiak, 1999; Taylor-Clarke et al., 2004; Weber, 1834/1996) and receptive field (RF) geometry (Longo and Haggard 2011). Other results, however, show that tactile distance is also modulated by higher-level factors, including tool use (Canzoneri et al., 2013; Miller et al., 2014), categorical segmentation of the body into discrete parts (de Vignemont et al. 2009; Knight et al., 2014), and illusions of body part size (Taylor-Clarke et al., 2004; de Vignemont et al. 2005; Tajadura Jimenez et al. 2012). Together, these results suggest that tactile distance perception is shaped by a combination of bottom-up and top-down factors. The neural bases of this ability, however, remain uncertain.

One possibility is that perceived distance may be a relatively direct readout of the structure of tactile space as coded by body maps in early somatosensory cortex. This interpretation is supported by the fact that tactile distance adaptation aftereffects show low-level characteristics such as orientation- and location-specificity (Calzolari et al., 2017), as well as by the relation between tactile distance illusions and factors such as cortical magnification and RF geometry. Tactile distance adaptation aftereffects occur following prolonged adaptation to a particular tactile distance, when subsequent distances are perceived as smaller on skin regions adapted to large distances, and vice versa (Calzolari et al., 2017). In this case, the representation of the body (i.e., hand) in the primary somatosensory cortex (SI) should mirror the distortions observed perceptually. An alternative possibility is that tactile distance may be calculated at higher-level processing stages such as for instance in the posterior parietal cortex at which distorted primary representations of the skin may be (at least partially) corrected, a form of tactile size constancy. For example, Huang and Sereno (2007) found that the overrepresentation of the lips relative to the rest of the face seen in SI maps is reduced in face maps in the ventral intraparietal area (VIP). This interpretation is supported by: (1) the fact that factors such as illusions of body size and tool use alter perceived tactile distance, which suggests that tactile distance perception is not a direct readout of low-level tactile processing, (2) the fact that while tactile distance illusions mirror distortions of somatotopic maps they are much smaller in magnitude than the latter (Taylor-Clarke et al., 2004; Longo 2017), and (3) the finding that disruption of processing in posterior parietal cortex with transcranial direct current stimulation (tDCS) impairs perception of tactile distance (Spitoni et al., 2013). Therefore, on this view, the representation of the body in posterior parietal cortex should mirror perception, whereas SI should show much larger distortions - i.e., greater anisotropy.

In this study we investigated the neural representation of tactile space by directly comparing neural and perceptual maps of the hand dorsum. We applied a method we recently developed to reconstruct perceptual configurations from the pattern of distance judgments using multidimensional scaling (MDS) (Longo and Golubova 2017). MDS is a method for reconstructing the latent spatial structure underlying a set of items given a matrix of pairwise distances, or dissimilarities, between items (Cox and Cox, 2001; Shepard, 1980; for a similar approach applied to neurophysiological data see Sereno and Lehky, 2011). Longo and Golubova (2017) obtained judgments of the distance between touches ap- plied to every pair of 16 locations arranged in a $4 \times 4$ grid on the hand dorsum. By applying MDS to the resulting perceptual distance matrix, they constructed perceptual maps of the skin which they then compared to actual skin shape. These configurations were clearly distorted, being elongated in the medio-lateral hand axis.

Here, we constructed neural maps of tactile space in an analogous manner. We used representational similarity analysis (Kriegeskorte et al., 2008a, 2008b) to investigate the structure of tactile space in different brain areas. By applying MDS to the representational dissimilarity matrix for a set of skin locations in a region of interest (ROI), we could reconstruct the neural representation of tactile space, and compare these configurations to the perceptual ones and to actual skin shape. We aimed to determine in which brain regions we could reconstruct maps of the skin from the distributed patterns of representational similarities. In addition, we aimed to measure the distortions of such maps to determined which brain areas show distortions most closely matching those seen perceptually: early primary cortices in line with hypothesis 1 , or later parietal regions in line with hypothesis 2 .

\section{Materials and methods}

\subsection{Participants}

The same twelve participants (mean $\pm \mathrm{SD}=29.5 \pm 6.3$ years; 6 females) participated both in the behavioural and fMRI experiments. Participants were neurologically intact and all but one were right-handed as assessed by the Edinburgh Handedness Inventory (Oldfield, 1971; $M=66$ range-90-100). All procedures were approved by the Department of Psychological Sciences Research Ethics Committee at Birkbeck, University of London. The study was conducted in accordance with the principles of the Declaration of Helsinki.

\subsection{Stimuli}

The MRI-compatible air-puff stimulator is shown in Fig. 2A (Dodecapus; Huang and Sereno, 2007). It was used to apply tactile stimulation on the dorsum of the participant's right hand (see Fig. 2B). The device was driven by an air compressor in the scanner control room which provided the input to a 9-way solenoid manifold valve ("S" Series Valve; Numatics Inc., Highland, MI) that was controlled by transistor-transistor logic pulses. Nine plastic air tubes from the manifold valve, for the fMRI experiment, passed through waveguides into the scanner room, where they connected to a block mounted beside the right hand, at the edge of the scanner's bore. The block served as a rigid base for 9 flexible tubes with nozzles (Loc-Line Inc., Lake Oswego, OR), flexibly arranged to direct $50 \mathrm{~ms}$ air puffs (input air-pressure $3.5 \mathrm{bar}$ ) at 9 locations arranged $2.5 \mathrm{~cm}$ apart on a $3 \times 3$ grid (Fig. $2 \mathrm{~A}$ ). The nozzles were approximately centred on the dorsum of the right hand over the locations that were marked with a felt pen using a customized stencil with punched holes on a $3 \times 3$ grid (Fig. 2 B). The tubes were never in contact with the skin surface. Each air puff was perceived as a localized and light touch on a corresponding hand dorsum location. To ensure that the stimulators were placed in the same position for the behavioural and imaging experiments the top central hole of the grid was positioned $2 \mathrm{~cm}$ below the knuckle of the middle finger in a direction of the wrist. The grid was aligned with the proximo-distal axis of the middle finger following an imaginary line connecting the fingertip and the knuckle of the middle finger.

\subsection{Procedure}

\subsubsection{Behavioural experiment}

Before the fMRI experiment, participants completed a behavioural experiment in which we asked them to estimate the distance between two touches on the dorsum of their hand. The rationale for this approach 
was twofold. First, we wanted to test the suitability and effectiveness of our paradigm using the air puff stimulator, which has not previously been used for tasks involving distance judgments. Second, we wanted to have an estimation of the perceptual configuration of the skin in each participant to compare with the neural data. Participants sat comfortably in front of a computer screen, with their right hand lying flat on the table, palm down, with the wrist straight. A black curtain occluded their right hand and forearm. On each trial, participants viewed a black screen and received two sequential air puff stimulations. Each stimulus lasted $450 \mathrm{~ms}$ with a $50 \mathrm{~ms}$ inter-stimulus interval between stimulation of the two locations.

Shortly after the second stimulus (jittered randomly between 1 and $2 \mathrm{~s}$ ), a line appeared at the centre of the screen. The participant was required to adjust the length of the line to match the perceived distance between the two tactile stimuli (for a similar procedure see Tamè et al., 2017). The initial length of the line ( 40 pixels $/ 1.54 \mathrm{~cm}$ or 460 pixels $/ 17.69 \mathrm{~cm}$ ) and the line orientation (horizontal or vertical) were counterbalanced within each block. Their order of presentation was random. Lines were approximately $1 \mathrm{~mm}$ thick and were white on a black background. Participants made un-speeded responses, adjusting the line length on the screen by pressing two arrow buttons on a keypad with the left hand. When they were satisfied with their response, they pressed a third button to confirm their response. Participants were never allowed to look at either hand during the experiment. An audio headset was used to present white noise to mask any acoustic cues from the air-puff stimulator.

There were four blocks with 72 trials each, for a total of 288 trials. In each block, there were 36 possible combinations of the 9 points, crossed with two orders of stimulation, which were presented in a random order. The 36 distances represent the unique possible pairs of the 9 stimulus locations. At the end of the experiment, a photograph was taken of the participant's right hand to calculate the actual size of the grid. A ruler appeared in the photographs allowing conversion between distances in pixels and $\mathrm{cm}$. Participants were allowed short breaks between blocks. The experimenter remained in the room throughout the session to ensure that participants complied with the instructions and to keep the position of the hand in place.

\subsection{2. fMRI experiment}

Participants lay in the scanner with their right hand prone outside the scanner bore, and wore earplugs throughout the experiment. The air-puff stimulators were positioned just over the dorsum of the participants' right hand as in the behavioural experiment by means of an fMRI compatible plastic plate to arrange the stimulators into a $3 \times 3$ grid suspended over the hand without touching the skin (as for the behavioural experiment; see the Results section and Fig. 2). At the beginning of each run, participants were instructed to close their eyes and focus their attention on the dorsum of their right hand. Air-puff stimuli were delivered sequentially in a random order on the different 9 points. Each run lasted about $11 \mathrm{~min}$ and included 55 trials. In each trial, the same point of the skin was stimulated by delivering the air quickly alternating between ON (50 ms) and OFF (50 ms), except for the oddball stimulation that was ON $(20 \mathrm{~ms})$ and OFF ( $80 \mathrm{~ms})$. The oddball stimulation was delivered to ensure that participants were focusing on their right hand as they were asked to report the number of oddball stimulations at the end of the run. There were four oddball trials per run, for a total of 16 trials in the whole experiment, the data was included in the analyses. Each point was stimulated 5 times in each run for a duration of $12 \mathrm{~s}$. In addition, 10 12-s trials of no stimulation (null trials) were randomly interleaved with the experimental trials.

\subsubsection{Stimulation and procedure for the functional localiser}

After the main experiment participants underwent a functional hand dorsum localizer. Somatosensory stimulation was applied to the dorsum of the right hand, the same location as for the main experiment. Stimulation was performed manually by the experimenter by brushing the par- ticipants' skin with a toothbrush across different directions - i.e., along the proximo-distal and medio-lateral axis, in a back-and-forth manner with a frequency of about $2 \mathrm{~Hz}$. This method has been previously successfully used and proved to be effective in evoking activity in the somatosensory cortices (Disbrow et al., 2000; Eickhoff et al., 2007). The paradigm consisted of 8 cycles each of them characterised by $16 \mathrm{~s}$ of stimulation and $16 \mathrm{~s}$ of rest. The localizer lasted overall $4 \mathrm{~min}$.

\section{4. fMRI data acquisition}

Echoplanar images $\left(2.33 \times 2.33 \mathrm{~mm}^{2}\right.$ in-plane, 2.3 -mm-thick slices, 662 volumes per run, 36 axial slices, flip $=90^{\circ}, \mathrm{TE}=39 \mathrm{~ms}, \mathrm{TR}=1 \mathrm{~s}$, $64 \times 64$ matrix, bandwidth $=1474 \mathrm{~Hz} /$ pixel, data acquired with prospective motion correction) were collected during 4 runs on a Siemens Avanto 1.5 T MRI scanner with a 32-channel head coil. For the functional localizer, echoplanar image parameters were the same as for the main experiment except for the number of volumes that were 256. For the anatomical image, we used an MPRAGE scan $(1 \times 1 \times 1 \mathrm{~mm}$, flip $=7^{\circ}$, TR $=1 \mathrm{~s}$, TI $=1 \mathrm{~s}$, TE $=3.57 \mathrm{~ms}$, matrix $256 \times 224 \times 176$ $190 \mathrm{~Hz} /$ pixel).

\section{5. $f M R I$ data preprocessing and GLM analysis}

Before analysis, the first eight volumes of the functional data of each run were discarded to avoid T1 saturation. The anatomical data were segmented using the standard procedure in FreeSurfer (function recon-all; Fischl et al., 1999), whereas the functional data were preprocessed and analyzed using Statistical Parametric Mapping software (SPM12; Wellcome Centre for Neuroimaging, University College London, London, UK; http://www.fil.ion.ucl.ac.uk/spm). Each functional volume was first bias-corrected and then spatially realigned to the first volume of the first run to correct for head movements. The functional volumes were then coregistered to the volumetric anatomical image which was aligned with the surfaces obtained from Freesurfer. First-level analyses were first carried out in the subject space and then the data were normalized to the Freesurfer common space (fsaverage). Data were spatially smoothed using a spatial Gaussian kernel of FWHM of $5 \mathrm{~mm}$ for the univariate second-level analyses only. The multivariate analyses were conducted using the unsmoothed data by means of the Matlab toolbox CoSMoMVPA (Oosterhof et al., 2016a) and in-house Matlab scripts.

For each voxel, we estimated the response to the stimulated points by fitting a general linear model (GLM) to the functional data. Each event was modelled using a square-wave function that was convolved with the canonical hemodynamic response. Therefore, for each run, the design matrix was 662 volumes X 9 predictors of interest. We also added six columns to account for head movements and one constant column. Note that the predictors coded the ON and OFF blocks. The GLM analysis returned 9 betas per run for each voxel, thus we obtained 36 betas of interests. The betas associated with the various points were averaged across runs and the resulted 9 averaged betas were used for the subsequent representational similarity analysis (RSA).

We also ran another similar GLM analysis using the smoothed data and the estimated betas were then used for the second-level univariate analyses. To this aim, we also estimated the betas and t maps associated with the contrast all stimulated points vs baseline that were subsequently used for the second-level analysis.

Data preprocessing steps for the functional localiser were identical to the ones performed for the main experiment. For the GLM a single event was modelled using a square-wave function that was convolved with the canonical hemodynamic response. The design matrix was 256 volumes $\mathrm{X} 1$ predictor of interest. We also added six columns to account for head movements and one constant column. The GLM analysis returned 1 beta for each voxel. 
Table 1

For each ROI, we show the minimum dimension (Min K) a cluster should have to be considered significant as resulting from the cluster-based

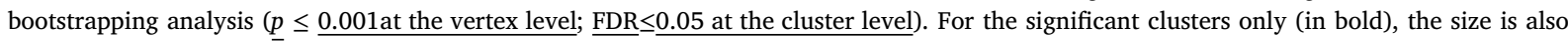
reported (rightmost column).

\begin{tabular}{|c|c|c|c|c|c|c|c|c|}
\hline Hemisphere & ROI & ROI size & Glasser's atlas & $\operatorname{Min} \mathrm{K}$ & $\begin{array}{l}\text { \# Significant } \\
\text { clusters }\end{array}$ & Cluster Size & $\mathrm{p}$-value & P crit (FDR \\
\hline \multicolumn{9}{|l|}{ Left } \\
\hline & SI & 9373 & $3 b, 1$ & 142 & 2 & 1921 & $<10^{-6}$ & 0.0108 \\
\hline & & & 2 & & & 197 & $<10^{-6}$ & 0.0108 \\
\hline & SII & 2327 & Op1, Op2-3, Op4 & 210 & 0 & & 0.4062 & 0.0014 \\
\hline & M1 & 4015 & 4 & 171 & 1 & 238 & $<10^{-6}$ & 0.0044 \\
\hline & $S P L$ & 2504 & $7 b / L I P$ & 239 & 0 & & 0.1788 & 0.0019 \\
\hline & $E V C$ & 4181 & $V 1, V 2, V 3$ & 98 & 0 & & 0.0654 & 0.0067 \\
\hline \multicolumn{9}{|l|}{ Right } \\
\hline & SI & 9280 & $3 b, 1,2$ & 88 & 0 & & 0.1102 & 0.0029 \\
\hline & SII & 2132 & Op1, Op2-3, Op4 & 196 & 0 & & 0.4064 & 0.0012 \\
\hline & M1 & 3855 & 4 & 238 & 0 & & 0.1864 & 0.0014 \\
\hline & $S P L$ & 2720 & $7 b / L I P$ & 257 & 0 & & 0.4440 & 0.0009 \\
\hline & $E V C$ & 478 & $V 1, V 2, V 3$ & 105 & 0 & & 0.1496 & 0.0107 \\
\hline
\end{tabular}

*Glasser et al., 2016.

\subsection{Region of interest identification and searchlight analysis}

We identified four regions of interest (SI; secondary somatosensory cortex, SII; primary motor cortex, M1; superior marginal gyrus, SPL) and one as a control (early visual cortex, EVC) on the basis of both anatomical and functional criteria (Dinstein et al., 2008; Cavina-Pratesi et al., 2010; Gallivan et al., 2011). To create the masks at the surface level, we used the recent multimodal cortical parcellation of the human brain developed by Glasser et al. (2016). First, we superimposed the functional localizer with the main experiment functional maps to determine the brain regions that were involved in tactile processing for both type of stimulations. Subsequently, we selected the vertices of interest for each participant based on Glasser and colleague's parcellation atlas at individual brain space. Specifically, our SI included areas 3a, 3b, 1 and 2 of Glasser and colleagues' nomenclature; SII included OP1, OP2-3 and OP4; M1 included area 4; SPL included 7 PC and AIP. We also analysed the early visual cortex including areas V1, V2 and V3 to assess the response pattern also in brain areas outside the typical tactile network. The mean average number of vertices across participants for each ROI are reported in Table 1.

For the fMRI experiment the main analysis is described in Fig. 1. We explored each ROI using a searchlight analysis (Kriegeskorte et al., $2006,2007)$ to identify areas that contained meaningful activity patterns about the spatial configuration - i.e. shape - of the skin of the dorsum of the right hand. Note that the searchlights do not have a typical geometrical shape (e.g. a sphere) because we used the estimated outer and inner cortices to select grey matter voxels only. In our approach (as implemented in the toolbox cosmomvpa), we selected the $\mathrm{N}$ closest neighbours of the central voxel, but in this case the resulting searchlight won't be a sphere as the selected voxels are not uniformly selected given the discrete nature of the data and low spatial resolution. As a result, our searchlights have an idiosyncratic shape containing grey matter voxels only. In a searchlight analysis, all voxels of interest are explored. At each step of the analysis, a voxel is selected with its neighbours (a searchlight; Fig. 1, step1) to obtain a multivariate representation (i.e., a pattern of neural responses; Fig. 1, step2) of each stimulated location. Each searchlight consisted of 100 voxels (the central voxel and its 99 closest neighbours within the ROI). We decided to select 100 voxels based on previous studies (Oosterhof et al., 2012; Proklova et al., 2016) and for practical reasons. In order to have a sufficient number of features for conducting the multivariate analysis but also not to cover a too large area along the surface because the hand dorsum is under-represented in the cortex compared to other neighbouring body parts (e.g. fingers). With this number of voxels, the averaged distance between a central voxel and its farthest neighbour of a searchlight was around $7.8 \mathrm{~mm}$.
As far as we know, there is no direct measurements of the hand dorsum representation in the human cortex, but there are studies that directly measured finger representations (e.g., Roux et al., 2018) that showed that, when averaging across participants, all fingers were represented within a surface patch of around $150 \mathrm{~mm}^{2}$.

For each searchlight (100 voxels), we had 9 neural patterns of betas (i.e., one for each of the stimulated locations; Fig. 1, step 2), from which we computed the 36 pairwise Euclidean distances (Fig. 1, step 3). We decided to use Euclidean distances rather than correlation coefficients, because it seemed a more appropriate measure to adopt in the present context, given that our purpose was to estimate spatial distances on the skin surface. We then used multidimensional scaling to estimate a 2-D neural configuration of the skin from this distance matrix (Fig. 1, step 4), analogous to the way we constructed a perceptual configuration from the matrix of judged distances in the behavioural experiment. Next, we applied Procrustes alignment (see next section) to obtain a measure of difference between this neural configuration and our model (i.e. the behavioural configuration; Fig. 1, step 5). Finally, the resulting Procrustes distance was projected onto the surface patch enclosing the central voxel of the current searchlight (Fig. 1, step 6). These steps were repeated for each voxel within each ROI. This analysis was conducted at the volume level (voxel-based) but using the outer and inner cortices obtained from Freesurfer as a constraint to select the voxels within each searchlight, as implemented in CoSMoMVPA (Oosterhof et al., 2011, 2016b). This allowed us to distinguish between regions that are adjacent on the surface (e.g., SI and M1). The searchlight was conducted in the subject space and the resulted maps were then resampled to a common space (the Freesurfer template fsaverage) for the group analysis.

\subsection{Multidimensional scaling}

Analysis procedures were similar to those in our previous study using this paradigm (Longo and Golubova 2017). The eight repetitions of each stimulus pair for an individual participant were averaged, resulting in a symmetric matrix reflecting the pairwise perceived distance between pairs of points, with zeros on the diagonal. Classical multidimensional scaling was applied to the distance matrix for each participant using the cmdscale command in MATLAB (Mathworks, Natick, MA). The output of MDS is a set of eigenvalues for each dimension and coordinates for each landmark in each dimension. As there are 9 landmarks, MDS attempts to position the landmarks in 9-dimensional space such that the distances between them are as proportional as possible to the perceived distances. To calculate the percentage of variance in the data accounted for by each dimension, we compared the absolute value of each eigenvalue to the sum of the absolute values of all 9 eigenvalues. The percentage variance 

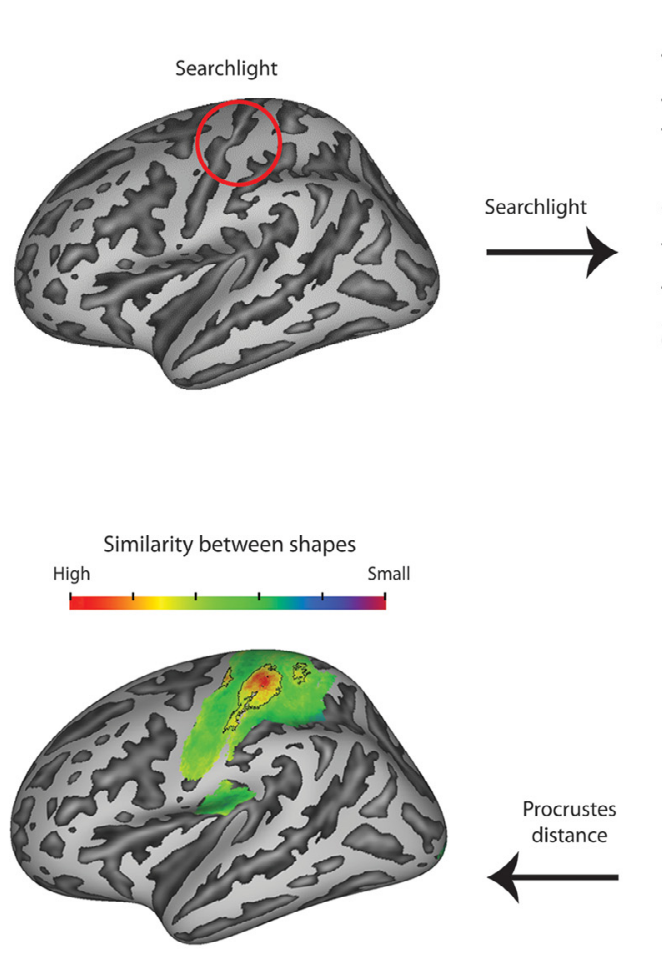
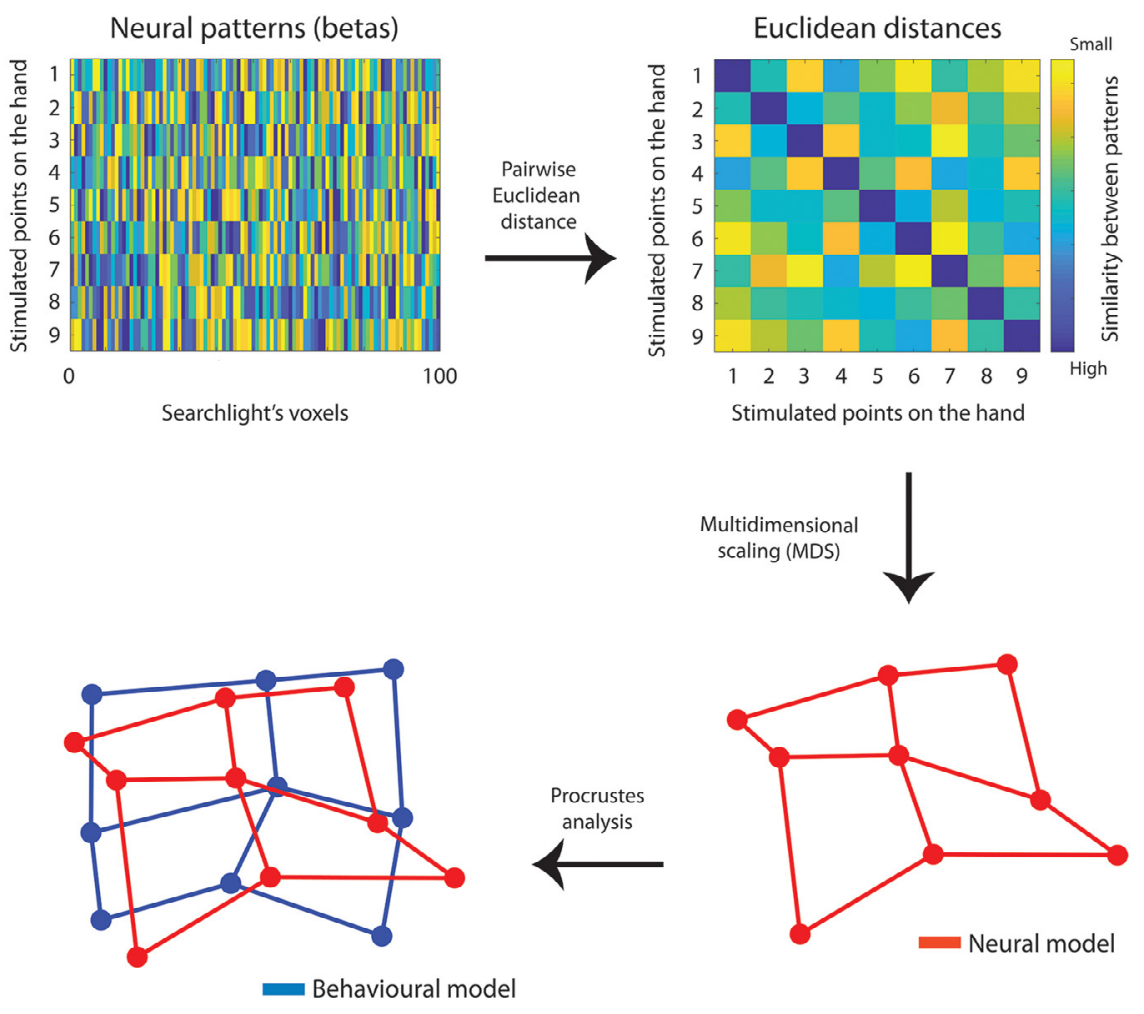

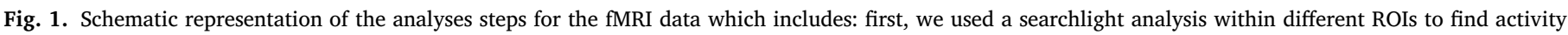

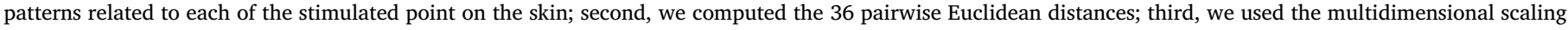

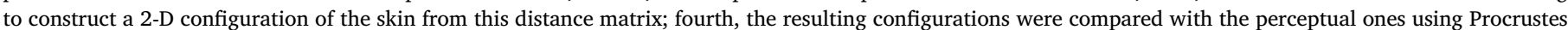

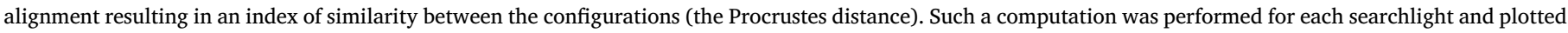
on the brain anatomy. Small Procrustes distances indicate greater similarity between the perceptual and neural configurations.

explained for the behavioural and neural data for the main ROIs are reported in Figure $\mathrm{S} 1$ of the supplementary materials.

For the behavioural data, to create a null distribution for comparison with our data, we conducted MDS on simulated random data following the procedure used in the study of Longo and Golubova (2017). For each simulation, 36 random numbers selected from a distribution evenly spaced between 0 and 1 were generated and placed into a distance matrix, as with the actual data. MDS was applied to each simulation and the eigenvalues and coordinates extracted. Then, the proportion of variance accounted for by the first two eigenvalues was calculated. One million such simulations were conducted.

For the fMRI experiment the main analysis is described in Fig. 1. For each searchlight (100 voxels), we had 9 neural patterns of betas (i.e., one for each of the stimulated locations), from which we computed the 36 pairwise Euclidean distances. We then used multidimensional scaling to construct a 2-D representational configuration of the skin from this distance matrix, analogous to the way we constructed a perceptual configuration from the matrix of judged distances in the behavioural experiment.

\subsection{Procrustes analyses}

Procrustes alignment (Rohlf and Slice 1990; Goodall 1991) superimposes two spatial configurations of homologous landmarks by translating, scaling, and rotating them to be as closely aligned as possible. First, the two configurations are translated so that their centroids (i.e., the centre of mass of all landmarks) are in the same location. Second, the configurations are normalized in size so that the centroid size, which is quantified as the square root of the sum of squared distances between each landmark and the centroid, is equal to 1 . Third, the configurations are rotated to minimize the sum of squared distance between pairs of homol- ogous landmarks. Note that in the present study mirror reflections of the configurations were allowed, though in other contexts this may not be desirable. At this point, the configurations are in the best possible spatial alignment, with all non-shape differences removed (Bookstein 1991). We used Procrustes alignment in two ways, both as a way to quantify dissimilarity in shape and as a visualization tool. First, the residual sum of squared distances between pairs of homologous landmarks which is not removed by Procrustes alignment provides a measure of the dissimilarity in shape between the two configurations, called the Procrustes Distance. If two configurations have exactly the same shape, they will lie on top of each other following Procrustes alignment and thus have a Procrustes distance of 0 . In contrast, two configurations with no shared spatial structure at all will have a Procrustes distance of 1 , given that the size normalization results in a total sum of squared variance within each configuration of exactly 1 . Second, Procrustes alignment provides a natural way to visually display configurations, making differences in shape clearly apparent. Given that we had to compare several hand configurations, we used generalized Procrustes analysis (GPA) using Shape (a MATLAB toolbox from Dr Simon Preston, freely available for download [https://www.maths.nottingham.ac.uk/personal/spp/shape.php] based on an algorithm originating from Gower, 1975; TenBerge, 1977).

For the fMRI experiment, the resulting shape from the matrix of neural distances (created using MDS) was compared with the grid obtained from the behavioural experiment (and to the actual grid on the hand; see Figure S6 in the supplementary material). More specifically, we placed the two configurations into Procrustes alignment and calculated the resulting Procrustes distance (i.e., the dissimilarity in shape of the two configurations). The resulting Procrustes distance and corresponding Procrustes coordinates were assigned to the central voxel of the searchlight. Finally, the brain maps of Procrustes distances and coordinates of each 


\section{A. Apparatus and stimuli}

Back to front view

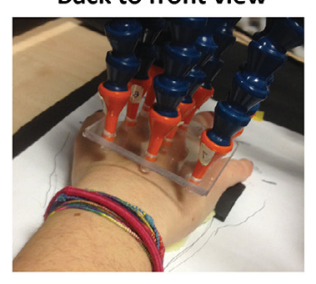

Lateral view
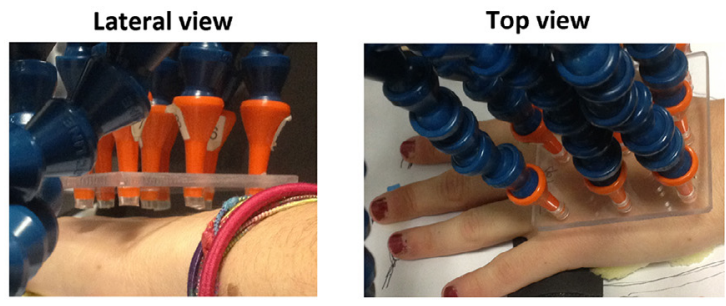

C. fMRI Paradigm

\section{B. Behavioural Paradigm}

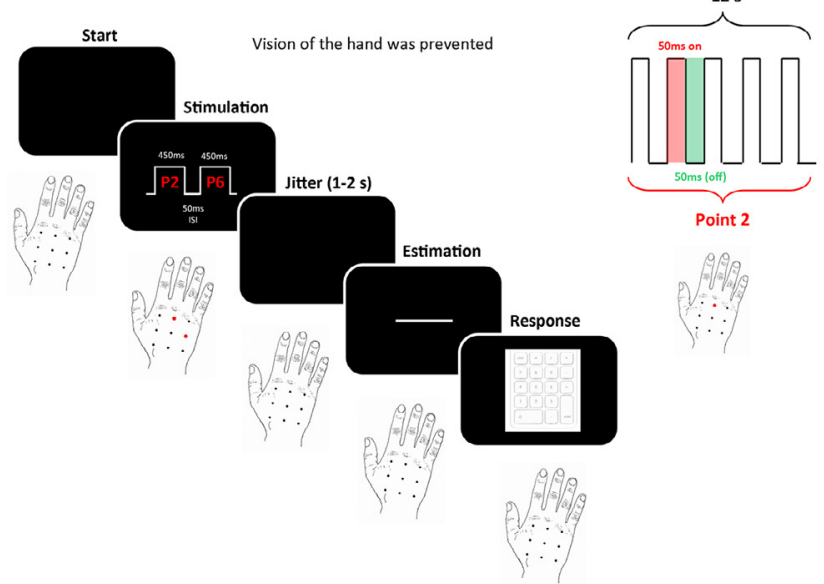

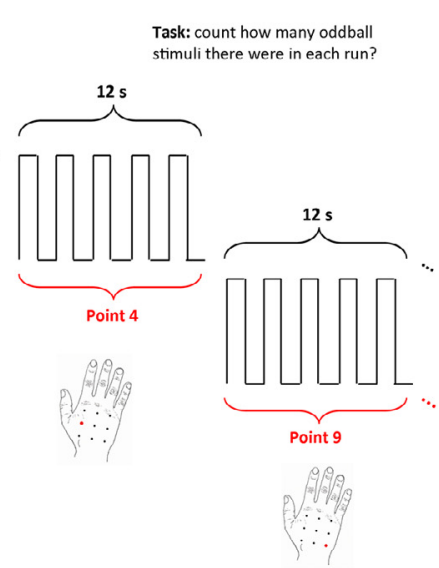

Fig. 2. Experimental paradigm. (A) Picture from the apparatus used to deliver air puff stimulation from different perspectives. The nine air puff nozzles were positioned on the top of the participant's right-hand dorsum and partially inserted into a plastic plate specifically designed to keep them in place forming a perfect square grid $(5 \times 5 \mathrm{~cm})$. The six air puff nozzles of the left and right sides of the plate were not perpendicular to the hand, but slightly tilted in the anti-clockwise (left) and clockwise (left) directions in order to resemble the curvature of the hand dorsum. Moreover, the top central nozzle was aligned with proximo-distal axis of the middle finger. Therefore, all the nozzles were positioned perpendicular to the skin surface. The nozzles were positioned at approximately $3 \mathrm{~mm}$ from the skin surface to prevent direct contact with it. The grid was aligned with the proximo-distal axis of the middle finger slightly clockwise rotated (i.e., away from the thumb). (B) Behavioural paradigm in which participants performed a tactile distance estimation task. In each trial participants looked at a black screen and received two sequential air puff stimulations. Each stimulus lasted $450 \mathrm{~ms}$ with a $50 \mathrm{~ms}$ inter-stimulus interval (ISI) between stimulation of the two locations (in the example location 2 and 6 stimulated are marked in red). After the second stimulus (jittered randomly between 1 and $2 \mathrm{~s}$ ), a line appeared at the centre of the screen. The participant was required to adjust the length of the

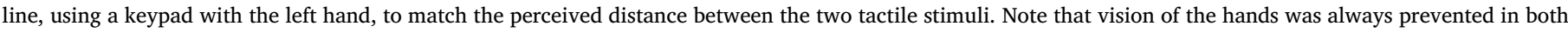

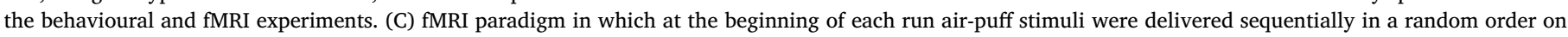

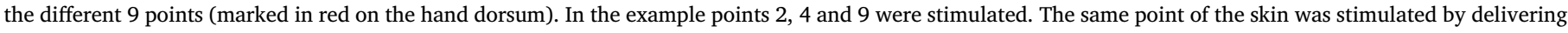

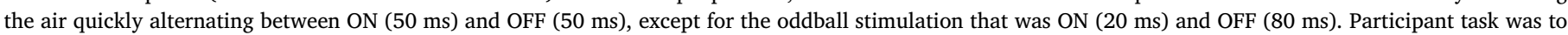
count how many oddball stimuli were delivered in each run. This was reported verbally to the experimenter at the end of each run.

participant were normalized to a common space (fsaverage) and averaged across participants. Note that because the Procrustes distance is a measure of dissimilarity, small numbers indicated that the similarity between the neural and the behavioural shapes was high.

The rationale for using the behavioural configurations was that these were the only representations that we knew existed in the brain since they were derived from the behavioural data. By contrast, the actual configurations, to the best of our knowledge, could only exist in the physical world. Indeed, it may be that such configurations are not present at all at the neural level. However, to assess the potential effect of the actual configurations, the same procedure was performed also using such shapes (see Figure S6 of the supplementary material for a comparison between the actual and behavioural configurations).

To evaluate which of the observed Procrustes distances were statistically smaller than chance, we ran a permutation analysis as described by Stelzer and colleagues (2013) to obtain a threshold size that a cluster (i.e. a set of neighbouring vertices) should have in order to be considered statistically significant (with $p<0.001$ at the vertex level and $p<0.05$ at the cluster level, as suggested by Stelzer et al., 2013). For each participant, we re-ran the same searchlight analysis as described above, but shuffling the 9 labels before computing the Euclidean distances and we repeated the procedure 100 times. We thus obtained 100 random Procrustes maps for each participant. We then carried out a bootstrap procedure to build a null distribution of averaged Procrustes distances: at each iteration, we randomly sampled (with replacement) one map from each participant's random Procrustes map and we then averaged across these 12 random maps. We repeated this procedure 10,000 times. Then, we computed a p-value at each vertex as the proportion of bootstrap samples that gave a Procrustes distance smaller than the actual Procrustes distance. We thus selected only those vertices that had a p-value smaller than 0.001 . Finally, we evaluated the threshold for a cluster to be statistically significant. We individuated neighbouring vertices that survived this threshold. We thus obtained a cluster size distribution. To evaluate the p-value associated with each cluster size, we divided the number of clusters for each size by the total number of clusters. The resulting pvalues were corrected using a false discovery rate (FDR) of 0.05 and the associated cluster size was used as threshold to select significant clusters in the observed data (see Table 1).

\subsection{Stretch estimation}

Moreover, we used the Procrustes distance, the sum-of-squares of the residual distances between pairs of homologous landmarks, as a measure of the dissimilarity between two configurations. This allowed us to estimate the overall stretch of perceptual configurations in the medio-lateral axis by finding the stretch applied to an idealized rectangular grid that minimized the dissimilarity with each configuration. We multiplied the $\mathrm{x}$-coordinates of a $3 \times 3$ square grid by a stretch parameter to generate grids of varying levels of stretch. When the stretch parameter was equal to 1 , the grid was perfectly square. When it was greater than 1 , the grid was stretched in the medio-lateral axis. When it was less than 1 , the grid was stretched in the proximo-distal axis. Note that because Procrustes alignment normalizes size, a stretch applied to the medio-lateral axis is identical to the inverse stretch being applied to the proximodistal axis. Thus, while distortions are described in terms of the mediolateral axis, this method cannot indicate which specific axis is affected by distortions in the sense that stretch of one axis is formally identical to compression of the other. For each participant, we determined the 
A

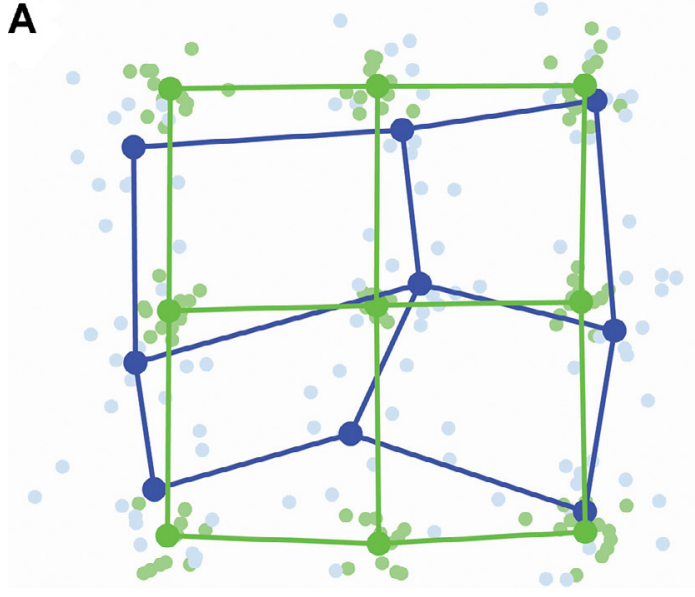

B

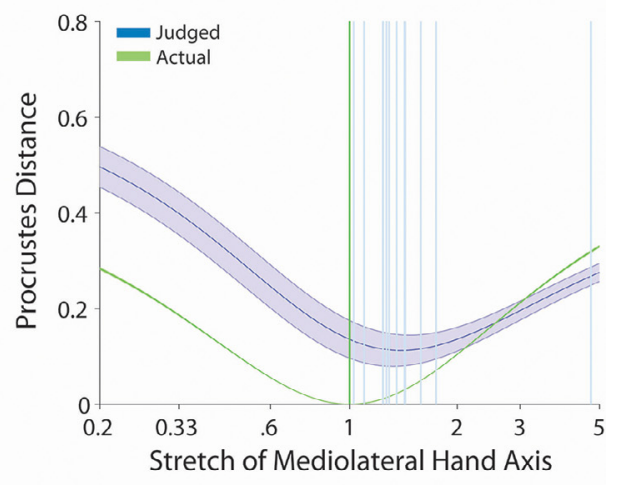

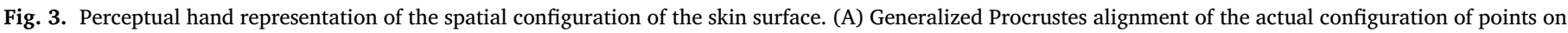

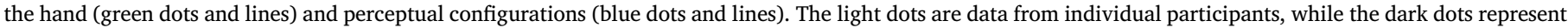

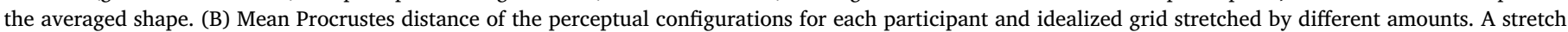

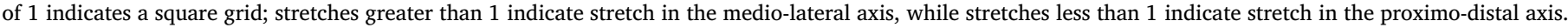
Blue lines represent values for each participant.

value of the stretch parameter that minimized the dissimilarity in shape (i.e., that minimized the Procrustes distance) between the stretched grid and the participant's perceptual configuration. Values between 0.2 and 5 were tested by exhaustive search with a resolution of 0.0005 units in natural logarithm space (i.e., 6438 steps). Note that we report mean stretch values as ratios, the statistical tests we report compare the mean logarithm of the ratios to 0 , since ratios are not symmetrical around 1 .

As a final step, we quantified the distortions of neural configurations at representational level within significant clusters adopting the same procedure described for the behavioural study: we extracted the shape associated to each significant cluster by averaging the Procrustes coordinates associated with the vertices within the cluster. Then, we stretched a square grid reflecting the locations of the 9 points by different amounts to find the stretch that minimized the Procrustes distance with each participant's neural configuration. As for the behavioural data, values between 0.2 and 5 were tested by exhaustive search with a resolution of 0.0005 units in natural logarithm space (i.e., 6438 steps).

\section{Results}

\subsection{Behavioural data}

The 2-dimensional perceptual maps of the skin as well as the actual configuration are shown in Fig. 3A (Individual data are shown in Figure S2 of the supplementary material). In order to quantify distortion of these configurations, we estimated the stretch applied to an idealized square $3 \times 3$ grid that minimized the dissimilarity with each configuration, as in previous studies (e.g., Longo and Golubova, 2017; Longo and Morcom, 2016).

Fig. 3B shows the mean Procrustes distance for values of the stretch parameter between 0.2 and 5 . The best-fitting stretch parameters were significantly greater than $1(M=1.47), \mathrm{t}(11)=3.38, p<0.006$, Cohen's $d=0.97$, indicating a substantial bias to overestimate distances in the medio-lateral compared to the proximo-distal hand axis. (Note that for this and other tests involving ratios, the calculation of means and all statistical tests were conducted on log-transformed values, which were converted back to ratios to report mean values). As predicted, this result replicates the anisotropy in tactile distance perception previously reported on the hand dorsum (Longo and Haggard 2011; Longo and Golubova 2017).

\section{2. $f M R I$ data}

Fig. 4 shows the topographic distribution of the resulting group Procrustes distances between the neural and perceptual configurations. Warm colours indicate small Procrustes distance, and thus high similarity between the shapes. Procrustes distances significantly smaller than chance (see Fig. 4B for the histograms of the Bootstrap values for the brain regions of the pre-defined ROIs in which we were able to reconstruct the geometry of the skin) and Figures S6 and S7 in the supplementary materials for the other brain regions) were found in clusters observed in contralateral SI (one in area $3 \mathrm{~b} / 1$ and another in area 2) and M1 (area 4) only, as shown in Fig. 4, Panel A and C (red contours indicate the significant clusters). Note that even though the searchlight was used to test five pre-defined ROIs, within SI it revealed three separate significant clusters. Therefore, these clusters have been named according to their location following the atlas of Glasser et al. (2016) (i.e., areas 3b/1, and 2). This distinction has been done post-hoc as we believe this was an important differentiation to highlight as these sub-regions are known to be structurally and functionally distinct (Kaas 1983a). Table 1 reports the outcome of the cluster analysis. No significant clusters were observed in the EVC or in the ipsilateral ROIs. Nearly identical results were obtained when we compared neural configurations to the actual grid shape (see Figure S6 of the supplementary materials). These results show that the perceptual structure of the skin can be reconstructed from the representational pattern in both primary somatosensory and motor cortices in the contralateral hemisphere. The shapes associated with each significant cluster are shown in Fig. 4D superimposed on the behavioural and actual shapes.

We investigated distortions at the level of neural representations using the same analysis of stretch applied to the behavioural data above. Fig. 4E shows the Procrustes distances as a function of the stretch for the perceptual, actual, and fMRI configurations (Procrustes distances as a function of the stretch for each individual participant are reported in Figures S3, S4 and S5 and all the ROIs in Figure S6 in the supplementary material).

Moreover, we also computed the Procrustes distance analysis at the whole brain level by performing a cluster-based bootstrapping analysis $(p<0.001$ at the vertex level; FDR $<0.05$ at the cluster level) on the whole brain to identify potentially significant clusters beyond the pre-defined ROIs. Such analysis confirmed the resulted significant clusters performed on the pre-specified ROIs, moreover, some other cluster 


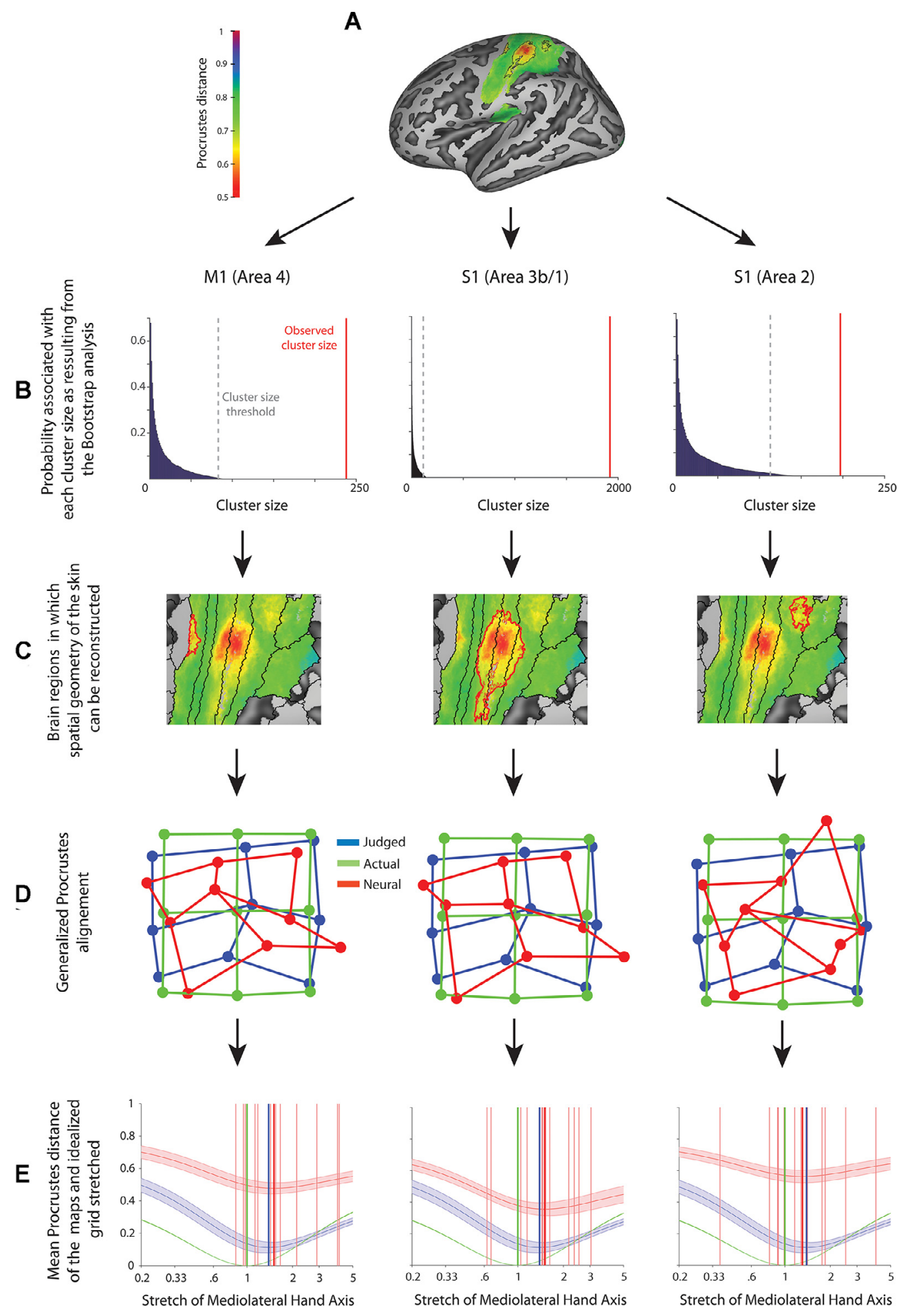

Fig. 4. (A) Brain regions in which the spatial geometry of the skin could be reconstructed from the representational pattern of neural activations. Red contours reflect significant cluster resulted from the cluster-based bootstrapping analysis ( $p<0.001$ at the vertex level; FDR $<0.05$ at the cluster level). (B) Probability associated with each cluster size as resulting from the Bootstrap analysis. The dotted grey lines represent the critical size values (cluster $\mathrm{p}$-value $<=0.05)$ for each ROI; the red lines represent the actual size of the observed cluster. Only the significant clusters are shown in this figure, refer to the supplementary Material for the other ROIs (C) Magnified view of the three significant clusters for area 4 (M1), area 3b/1 (SI) and area 2 (SI), respectively, when comparing the neural and perceptual configurations. The red colour represents the voxels in which the reconstructed representations were better achieved as expressed in Procrustes distance value. (D) Generalized Procrustes alignment of the grand average shape across participants of the actual configuration of points on the dorsum of the right hand (green dots and lines), perceptual (blue dots and lines) and neural (red dots and lines) configurations. (E) Mean Procrustes distance between behavioural (blue), fMRI (red: for each participant) and actual (green) grid on the participants' hand dorsum and idealized grids stretched by different amounts. A stretch of 1 indicates a square grid; stretches greater than 1 indicate stretch in the medio-lateral axis, while stretches less than 1 indicate stretch in the proximo-distal axis. The shaded regions indicate one standard error of the mean. The dotted vertical lines indicate the mean of the best-fitting stretches for fMRI (red) and behavioural configurations (blue). The stretch that minimized the Procrustes distance was substantially larger than 1 . Thus, there was clear evidence for stretch in the medio-lateral hand axis for perceptual configurations. 
resulted to be significant (see Figure S8 in the supplementary material), namely in the contralateral hemisphere Area 55b and OP4 (SII) and in the ipsilateral hemisphere what we define as the parietal operculum (PO) (note that the PO cluster was positioned in the straddling areas 2, PFt and PFop) and the Superior Temporal Visual area (STV).

Despite the presence of additional significant clusters, the quality of the reconstruction of the spatial geometry was not satisfactory for such clusters (Figure S9 in the supplementary material). However, despite its lower Procrustes distance value the reconstructed configuration for area PO was good resembling the shape of the original undistorted grid. Note that we have two uses of the Procrustes Distance. One is to quantify how well MDS reconstructs the true spatial structure of the skin. This value represents how different two shapes are, namely the smaller the Procrustes distance value the more similar are the two shapes. However, this value does not tell anything about whether the shape is distorted along a certain direction/axis. Therefore, the second use is to quantify the magnitude of stretch in each map. In this case, it is not small Procrustes distances per se that indicate distortion in the maps, but the fact that the stretch that minimizes the Procrustes distance is systematically different from 1 (i.e., Fig. 1E).

As shown in Fig. 4E, best-fitting values of the stretch parameter were significantly greater than 1 for the cluster in SI straddling areas $3 \mathrm{~b}$ and 1 (M: 1.47), $\mathrm{t}(11)=2.68, p=0.021, d=0.77$, and the cluster in M1 (M: $1.64), \mathrm{t}(11)=3.14, p=0.010, d=0.91$. However, for the cluster within area 2 of SI this distortion was not significant (M: 1.26), $\mathrm{t}(11)=1.33$, $p=0.212, d=0.38$. The stretch parameters for S1 (3b/1) and M1 significantly greater than 1 demonstrate that the grids are stretched in the medio-lateral axis. Note that such peculiar distortion that matched the perceptual one was specific for the sensorimotor regions. Moreover, none of these clusters showed a significant stretch parameter different from those observed for the perceptual configurations given by the behavioural data (all $p>0.45$ ). This shows that the neural data for these two clusters match the configuration based on participants' judgments of the tactile distance. Virtually identical results were obtained for the clusters identified by comparing neural configurations to actual skin configuration shape (see Figure S6 in the supplementary materials). Finally, we did not find a significant subject-by-subject correlation for the stretch parameters when comparing the behavioural data with the neural data for S1 and M1. This can be ascribed to the noise in the fMRI data or to some extent to the small sample size.

\section{Discussion}

In the present study, we reconstructed the internal geometry of tactile space using representational similarity of neural patterns between locations on the skin. Behaviourally, we replicated previous reports that tactile space is stretched along the medio-lateral axis of the hand dorsum (e.g., Longo and Golubova, 2017; Longo and Haggard, 2010, 2012). Critically, using a novel approach that combine fMRI with MDS, we showed that similar distortions can be measured directly from neural data. Strikingly, this was evident in the primary sensorimotor cortices contralateral to the locus of stimulation. Therefore, these low-level cortical brain areas carry information corresponding to the distorted perceptual structure of tactile space of the hand dorsum being stretched along the medio-lateral axis.

Interestingly, the sensorimotor cortices were the only brain areas from which we were able to reconstruct maps of the shape of the skin. Previous studies have shown the presence of clear somatotopically organized representations of different body parts in SI contralateral to the locus of stimulation (e.g., Huang et al., 2012; Sanchez-Panchuelo et al., 2010). However, this does not seem to be the case for the hand dorsum, in which, to the best of our knowledge, clear maps have not been shown in humans. Recently, a study has shown only a difference in terms of peak of cortical activation and numbers of activated voxels between the dorsum and the palm of the hand, with the former being lower than the latter (Jang et al., 2013). Moreover, in the monkey neurophysiological literature, it is unclear to what extent similar topographic maps (i.e., palm and dorsum of the hand) can be clearly defined, given that these neurophysiological studies have shown that representations of the dorsal hand surface may fall outside the global somatotopic pattern in SI (Kaas 1983b).

The distortions of the neural maps we constructed from representational similarity of neural patterns in contralateral sensorimotor cortex provide an intriguing correspondence with the anisotropic geometry of (RFs) in the somatosensory cortex (Brooks et al., 1961; Alloway et al., 1989). We have proposed that tactile space can be thought of as a 2dimensional array in which the RFs of neurons in somatotopic maps forming the "pixels" of the grid (Longo and Haggard 2011; Longo 2017; Fiori and Longo 2018). Where RFs differ in size on different skin surfaces, this will produce a perceptual magnification on the surface with smaller RFs, assuming that RF overlap is comparable (that is, assuming that regions with smaller RFs occupy proportionally more cortical area). Neurophysiological studies have provided some evidence for this assumption, finding that overlap between the RFs of adjacent neurons is a constant proportion of RF size across a wide range of sizes (Sur et al., 1980). Where individual RFs are anisotropic (e.g., ovalshaped), this will produce a perceptual stretch along the shorter axis of the RF. The somatosensory RFs on the hairy skin of the limbs tend to be oval-shaped with the long-axis aligned with the proximo-distal limb axis (e.g., Brooks et al., 1961; Alloway et al., 1989), compatible with the results of the present study. However, the magnitude of these distortions is much smaller than what would be predicted only on the basis of differences in RF size and shape. Indeed, the long axis of RFs in somatosensory cortex is frequently $4-5$ times the length of the small axis (e.g., Brooks et al., 1961), yet the magnitude of perceptual anisotropy is again only a small fraction of that (e.g., Green, 1982; Longo and Golubova, 2017; Longo and Haggard, 2011b). We suggest that a process of tactile size constancy which corrects for distortions inherent in primary representations to produce (approximately) veridical percepts of size may take place in the sensorimotor cortices, particularly, in the primary somatosensory cortex where the reconstructed skin shape was more accurate. It is interesting to note that this bias is reduced or even eliminated on the glabrous skin of the palm (Longo and Haggard 2011; Knight et al., 2014; Longo et al., 2015a; Longo 2020). This is most likely due to the fact that RFs on the glabrous skin are generally more circular and, when oval-shaped, do not tend to have a preferred orientation (e.g., DiCarlo et al., 1998; DiCarlo and Johnson 2002). Moreover, as suggested by Longo (2017) such anisotropies are not limited to the hand, but have been found on other body parts, including the forearm (Green, 1982; Le Cornu Knight et al., 2014), the leg (Green, 1982), and the face (Longo et al., 2015), suggesting that it may reflect a basic principle of body representation, rather than something idiosyncratic to the hands, or even to limbs.

In agreement with our results, a recent study using repetitive Transcranial Magnetic Stimulation (rTMS), have shown that the metric representation of the body depends on somatosensory afferences. In their study Giurgola et al. (2019) applied rTMS on the somatosensory cortices of both hemispheres representing the hands (i.e., SI) while participants judge whether visually presented right and left hands matched the size of their own hand. They found that rTMS produces distortions of the perceived size of the participants' own hand, but not other body parts (Giurgola et al., 2019). Intriguingly, this effect was not present when rTMS was applied on the inferior temporal parietal lobe, an area largely linked with body representation disturbances (Bolognini and Miniussi 2018). However, our approach did not allow us to rule out possible top-down interactions between SI and other brain areas (e.g., higher level regions), thus preventing any definite conclusion about the pathway leading to our results. Indeed, other brain areas may have interacted with the primary somatosensory cortex (and/or primary motor cortex) providing information to correct for homuncular distortions. It would be relevant to assess this question in a dedicated study which possibly involve other neuroimaging techniques with a higher temporal 
resolution than fMRI such as electroencephalography (EEG) or magnetoencephalography (MEG).

These results support the notion that the distance between tactile points on the skin of the hand dorsum is computed at a low cortical level of tactile representation processing. In this respect, Calzolari et al. (2017) using a tactile adaptation aftereffect paradigm suggested that tactile distance perception is a basic somatosensory feature supporting the idea that distance perception arises at relatively early stages in tactile processing. In their study, the authors explored how adaptation to a distance between two separate points, passively delivered on the hand dorsum, affects perception of subsequent distances. They found tactile distance aftereffects with passive touch. Moreover, their effect was orientation and region specific, did not transfer within and between the hands, and was encoded using skin-based coordinates. These are all features that point to a low level processing locus for tactile distance computation. Similarly in vision, Sperandio et al. (2012) found that the retinotopic activity in the primary visual cortex (V1) reflects the perceived rather than the retinal size of an afterimage. The fact that SI is critically involved in a complex processing such as tactile distance estimation is in accordance with literature showing that this low cortical level area may not be critical for performing simple tactile tasks - i.e., tactile detection - both in monkeys (LaMotte and Mountcastle 1979) and humans (Tamè and Holmes 2016). By contrast, SI seems to be critically involved in processing that were thought to be accomplished by higher level cortical areas, such as bilateral integration of touch (Tamè et al., 2012, 2016, 2015b) as well as tactile working memory (Harris et al., 2002; Katus et al., 2015).

Other behavioural studies that used a different paradigm which investigated participants' abilities to localize the position of the different parts of the hand relative to each other showed the presence of similar distortions. In this respect, Longo and Haggard (Longo and Haggard 2010,2012 ) asked participants to place their hand flat on a table underneath an occluding board and to use a long baton to judge the perceived location of the tip and knuckle of each of their finger. By comparing the relative location of judgments of each landmark, authors constructed perceptual configurations of hand structure which they then compared to actual hand form. A highly consistent pattern of distortions was apparent across participants, including overestimation of hand width, and underestimation of finger length. Longo et al. (2015b) conducted a similar study, but asked participants to judge the location of tactile stimuli applied to the hand dorsum, finding overestimation of distances in the medio-lateral hand axis, compared to the proximo-distal axis. Interestingly, this pattern of distortions is quite similar to that described in the present study. Therefore, the present results further support the idea that similar mechanisms may underlie body position sense and tactile distance perception (Longo and Haggard 2010). However, a recent study by Longo and Morcom (2016) has shown that there is no correlation between the magnitudes of distortion in body representation deriving from a position sense and tactile distance tasks. Authors suggest that this casts doubt on the proposal that a common body model underlies both tactile distance perception and position sense. It is important to note that in the present study for both the behavioural and fMRI experiments we adopted tasks that involved tactile localization and not position sense.

The fact that we were able to reconstruct the shape of the skin space based on activation elicited by tactile points both in the primary somatosensory and motor cortices suggests that M1 is also involved in the processing of the tactile stimuli. In everyday life, tactile stimulation is commonly accompanied or caused by action. Indeed, the sensory and motor systems are intimately related, both anatomically and functionally, with continuous reciprocal exchange of information (Rossi et al., 1998; Brochier et al., 1999; Nelson et al., 2004). These systems communicate via a network of extensive connections between the sensory and motor cortices (Asanuma et al., 1968; Strick and Preston 1982; Stepniewska et al., 1993; Andersson 1995; Huffman 2001; Makris et al., 2005; Shinoura et al., 2005; Eickhoff et al., 2010; Mao et al., 2011), but also by motor cortex cells responding directly to sensory stimuli, perhaps via their direct inputs from the dorsal column nuclei via the ventrolateral thalamic nucleus (Albe-Fessard and Liebeskind 1966; Goldring and Ratcheson 1972; Fetz et al., 1980; Fromm et al., 1984) and vice-versa (Matyas et al., 2010). The existence of direct connections between the sensory areas in the post-central gyrus and the motor areas of the precentral gyrus in humans has been recently demonstrated by Catani and colleagues who, using diffusion tractography, confirmed the presence of Ushape fibres that directly connect SI with the motor cortex (Catani et al., 2012), as previously demonstrated in invasive studies in animals. These fibers are thought to connect the somatosensory and motor areas of the cortical regions that are involved in the control of finely tuned movements and complex motor skills (i.e. the hand's brain regions). In this respect, Tamè et al. (2015a) combined tactile repetition suppression with the techniques of afferent inhibition (i.e., corticospinal excitability is inhibited when a single tactile stimulus is presented before a TMS pulse over the motor cortex) to investigate whether the modulation of somatosensory activity induced by double tactile stimulation propagates to motor cortex and alters corticospinal excitability in humans. They found that activity in the somatosensory cortices following repetitive (i.e., double) tactile stimulation also elicits finger-specific activation in the primary motor cortex demonstrating that spatial information is retained in the SI and then transferred to the motor cortex (Tamè et al., 2015a). Furthermore, the relation between the sensory and motor systems is particularly important in haptic tasks, in which we actively explore an object. In this situation, our brain is simultaneously receiving sensory signals from, and generating motor signals for, the movements. These inputs have to be combined to perceive and actively explored objects. In this respect, Ejaz et al. (2015), analysing activity patterns during individual fingers movements using fMRI, showed that hand use can shape fingers' arrangement in both the sensory and motor cortices (Ejaz et al., 2015).

It is also worth noting the substantial differences in the way that perceptual and neural maps of the skin were measured. Perceptual maps were based on explicit judgments of the distance between two stimulated locations on the skin. Neural maps, in contrast, were based on parameter estimates in each voxel for each location individually. MDS for neural maps was based on Euclidean distances between the patterns across voxels for each pair of stimulated locations, but this did not involve these pairs being stimulated on the same trials, as in the perceptual task. Indeed, in the neural task, the participant's task had nothing to do with distance at all. These differences were a result of the different requirements of psychophysical testing on the one hand, and RSA analysis of fMRI data on the other. It is notable that despite these differences, the spatial distortions of perceptual and neural maps were nevertheless strikingly similar. This suggests that the distortions seen in tactile distance judgments are not idiosyncratic to the act of judging distance, but reflect more general aspects of the organisation of tactile representations.

We have discussed our results as reflecting representation of the spatial location of touch on the skin. It is possible, however, that other frames of reference may be involved. As the position of the stimulated hand was kept constant throughout the experiment, spatial location on the hand was confounded with spatial location in an external egocentric frame of reference centred on the participant's body. Effects such as the crossed-hands deficit (Yamamoto and Kitazawa 2001) show that touch is automatically converted into external frames of reference, and studies measuring the time-course of this remapping show that it happens extremely rapidly (Azañón and Soto-Faraco 2008). In our view, it is most likely that the effects we find reflect spatial representation in a skin-centred frame of reference given that modulation of tactile responses by limb position in space has generally been found in more posterior regions of the parietal cortex than SI, both in fMRI studies in humans (Lloyd et al., 2003) and neurophysiological studies in monkeys (Sakata et al., 1973).

Finally, regarding the reconstructed configuration of the tactile space that emerged from the whole brain analysis in the ipsilateral parietal 
operculum (Figure S9 in the supplementary material) we do not have a definitive interpretation given that this was an unexpected result. A possibility could be that such area is actually carrying information about the actual configuration of the skin space, however, such result should be treated with caution given that despite the satisfactory reconstruction, the Procrustes value which represents the difference between the neural and behavioural shapes, was higher than every other brain area.

\section{Potential limitations}

The fMRI testing has been performed on a $1.5 \mathrm{~T}$ scanner which may be considered a limitation in terms of spatial resolution. However, there are recent studies that have been successfully performed using the same type of scanner that produced high quality resolution sensory maps (e.g., Sood and Sereno 2016; Carey et al., 2017). Moreover, recently Morgan and Schwarzkopf (2020) compared population receptive field (pRF) analysis maps acquired in the same three individuals using comparable scanning parameters on a 1.5 (the same scanner that we used in the present study) and a 3 Tesla scanner located in two different countries. They found that the signal-to-noise ratio for the 3 Tesla data was superior; critically, however, estimates of pRF size and cortical magnification did not reveal any systematic differences between the sites. Moreover, there was no substantial increase in the number of voxels containing meaningful retinotopic signals after low-pass filtering. This corroborates the notion that the potential limitation due to the scanner spatial resolution can be considered generally limited.

\section{Conclusion}

In the present study, by applying an innovative approach that combined MDS and Procrustes alignment on fMRI data, we were able to reconstruct the shape of the internal geometry of the skin of the hand dorsum. We showed that the superficial structure of the skin can be reconstructed from the matrix of perceived tactile stimulated points on the hand. Intriguingly, the reconstructed shape of the skin in the primary somatosensory and motor cortices matches the distortions that emerge at behavioural level (i.e., perceptual configurations) providing evidence that sensory-motor cortices may be a primary neural basis of such representations. Intriguingly, the sensorimotor cortices were the only regions that contained sufficiently coherent information to allow a satisfactory reconstruction of the shape of the skin space; we found nothing similar in data from higher level brain regions. We suggest that representations in SI and M1 are likely to be critical for haptic control (Johansson and Flanagan 2009) of complex hand-object interactions involving events that are precisely localized in space.

\section{Credit author statement}

Luigi Tamè: Conceptualization, Methodology, Validation, Formal analysis, Investigation, Writing - original draft, Visualization; Raffaele Tucciarelli: Validation, Formal analysis, Visualization, Writing - review \& editing. Renata Sadibolova: Investigation, Writing - review \& editing. Marty Sereno: Conceptualization, Methodology, Software, Validation, Formal analysis, Investigation, Writing - review \& editing; Matthew R. Longo: Conceptualization, Methodology, Validation, Formal analysis, Resources, Writing - review \& editing, Funding acquisition.

\section{Acknowledgement}

LT, RT and MRL were supported by a grant from the European Research Council (ERC-2013-StG-336050) under the FP7 to MRL.

\section{Supplementary materials}

Supplementary material associated with this article can be found, in the online version, at doi:10.1016/j.neuroimage.2021.117730.

\section{Reference}

Albe-Fessard, D., Liebeskind, J, 1966. Origine des messages somato-sensitifs activant les cellules du cortex moteur chez le singe. Exp. Brain Res. 1, 127-146.

Alloway, K.D., Rosenthal, P., Burton, H., 1989. Quantitative measurements of receptive field changes during antagonism of GABAergic transmission in primary somatosensory cortex of cats. Exp. Brain Res. 78, 514-532.

Andersson, G., 1995. Cortico-cortical mediation of short-latency (lemniscal) sensory input to the motor cortex in deeply pentobarbitone anaesthetized cats. Acta Physiol. Scand. 153, 381-392.

Asanuma, H., Stoney, S.D., Abzug, C., 1968. Relationship between afferent input and motor outflow in cat motorsensory cortex. J. Neurophysiol. 31, 670-681.

Azañón, E., Soto-Faraco, S., 2008. Changing reference frames during the encoding of tactile events. Curr. Biol. 18, 1044-1049.

Bolognini, N., Miniussi, C., 2018. Noninvasive brain stimulation of the parietal lobe for improving neurologic, neuropsychologic, and neuropsychiatric deficits. Handb Clin Neurol 151, 427-446.

Bookstein, F.L., 1991. Morphometric Tools For Landmark data: Geometry and Biology. Cambridge University Press, Cambridge, UK.

Brochier, T., Boudreau, M.-.J., Paré, M., Smith, A.M., 1999. The effects of muscimol inactivation of small regions of motor and somatosensory cortex on independent finger movements and force control in the precision grip. Exp. Brain Res. 128, 31-40.

Brooks, V.B., Rudomin, P., Slayman, C.L., 1961. Peripheral receptive fields of neurons in the cat's cerebral cortex. J. Neurophysiol. 24, 302-325.

Brown, P.B., Fuchs, J.L., Tapper, D.N., 1975. Parametric studies of dorsal horn neurons responding to tactile stimulation. J. Neurophysiol. 38, 19-25.

Calzolari, E., Azañón, E., Danvers, M., Vallar, G., Longo, M.R., 2017. Adaptation aftereffects reveal that tactile distance is a basic somatosensory feature. Proc. Natl. Acad. Sci. USA $114,4555-4560$.

Canzoneri, E., Ubaldi, S., Rastelli, V., Finisguerra, A., Bassolino, M., Serino, A., 2013. Tool-use reshapes the boundaries of body and peripersonal space representations. Exp. Brain Res. 228, 25-42.

Carey, D., Krishnan, S., Callaghan, M.F., Sereno, M.I., Dick, F., 2017. Functional and quantitative MRI mapping of somatomotor representations of human supralaryngeal vocal tract. Cereb. Cortex 27, 265-278.

Catani, M., Dell'acqua, F., Vergani, F., Malik, F., Hodge, H., Roy, P., Valabregue, R., de Schotten, M, 2012. Short frontal lobe connections of the human brain. Cortex 48, 273-291.

Cavina-Pratesi, C., Monaco, S., Fattori, P., Galletti, C., McAdam, T.D., Quinlan, D.J., Goodale, M.A., Culham, J.C., 2010. Functional magnetic resonance imaging reveals the neural substrates of arm transport and grip formation in reach-to-grasp actions in humans. J. Neurosci. 30, 10306-10323.

Cholewiak, R.W., 1999. The perception of tactile distance: influences of body site, space, and time. Perception. 28, 851-875.

Cox, T.F., Cox, M.A.A., 2001. Multidimensional Scaling, 2nd ed. Chapman and Hall, London.

de Vignemont, F., Ehrsson, H.H., Haggard, P., 2005. Bodily illusions modulate tactile perception. Curr. Biol. 15, 1286-1290.

de Vignemont, F., Majid, A., Jola, C., Haggard, P., 2009. Segmenting the body into parts: evidence from biases in tactile perception. Q. J. Exp. Psychol. 62, 500-512.

DiCarlo, J.J., KO, Johnson, 2002. Receptive field structure in cortical area 3b of the alert monkey. Behav. Brain Res. 135, 167-178.

DiCarlo, J.J., Johnson, K.O., Hsiao, S.S., 1998. Structure of receptive fields in area 3b of primary somatosensory cortex in the alert monkey. J. Neurosci. 18, 2626-2645.

Dinstein, I., Gardner, J.L., Jazayeri, M., Heeger, D.J., 2008. Executed and observed movements have different distributed representations in human aIPS. J. Neurosci. 28, 11231-11239.

Disbrow, E., Roberts, T., Krubitzer, L., 2000. Somatotopic organization of cortical fields in the lateral sulcus of Homo sapiens: evidence for SII and PV. J. Comp. Neurol. 418, $1-21$.

Eickhoff, S.B., Grefkes, C., Zilles, K., Fink, G.R., 2007. The somatotopic organization of cytoarchitectonic areas on the human parietal operculum. Cereb. Cortex 17, 1800-1811.

Eickhoff, S.B., Jbabdi, S., Caspers, S., Laird, A.R., Fox, P.T., Zilles, K., Behrens, T.E.J., 2010. Anatomical and functional connectivity of cytoarchitectonic areas within the human parietal operculum. J. Neurosci. 30, 6409-6421.

Ejaz, N., Hamada, M., Diedrichsen, J., 2015. Hand use predicts the structure of representations in sensorimotor cortex. Nat. Neurosci. 18, 1034-1040.

Fetz, E.E., Finocchio, D.V., Baker, M.A., Soso, M.J., 1980. Sensory and motor responses of precentral cortex cells during comparable passive and active joint movements. J. Neurophysiol. 43, 1070-1089.

Fiori, F., Longo, M.R., 2018. Tactile distance illusions reflect a coherent stretch of tactile space. Proc. Natl. Acad. Sci. USA 115, 1238-1243.

Fischl, B., Sereno, M.I., Dale, A.M., 1999. Cortical surface-based analysis. II: inflation, flattening, and a surface-based coordinate system. Neuroimage 9, 195-207.

Fromm, C., Wise, S.P., Evarts, E.V., 1984. Sensory response properties of pyramidal tract neurons in the precentral motor cortex and postcentral gyrus of the rhesus monkey. Exp. Brain Res. 54, 177-185.

Gallivan, J.P., McLean, D.A., Valyear, K.F., Pettypiece, C.E., Culham, J.C., 2011. Decoding action intentions from preparatory brain activity in human parieto-frontal networks. J. Neurosci. 31, 9599-9610.

Giurgola, S., Pisoni, A., Maravita, A., Vallar, G., Bolognini, N., 2019. Somatosensory Cortical Representation of the Body Size. Hum Brain Mapp hbm.24614.

Glasser, M.F., Coalson, T.S., Robinson, E.C., Hacker, C.D., Harwell, J., Yacoub, E., Ugurbil, K., Andersson, J., Beckmann, C.F., Jenkinson, M., Smith, S.M., Van Essen, D.C., 2016. A multi-modal parcellation of human cerebral cortex. Nature 536, 171-178. 
Goldring, S., Ratcheson, R., 1972. Human motor cortex: sensory input data from single neuron recordings. Science (80-) 175, 1493-1495.

Goodall, C., 1991. Procrustes methods in the statistical analysis of shape. J. R. Stat. Soc. Ser. B 53, 285-321.

Gower, J.C., 1975. Generalized procrustes analysis. Psychometrika 40, 33-51.

Green, B.G., 1982. The perception of distance and location for dual tactile pressures. Percept. Psychophys. 31, 315-323.

Harris, J.A., Miniussi, C., Harris, I.M., Diamond, M.E., 2002. Transient storage of a tactile memory trace in primary somatosensory cortex. J. Neurosci. 22, 8720-8725.

Huang, R.-.S., Chen, C., Tran, A.T., Holstein, K.L., Sereno, M.I., 2012. Mapping multisensory parietal face and body areas in humans. Proc. Natl. Acad. Sci. USA 109, 18114-18119.

Huang, R.-.S., Sereno, M.I., 2007. Dodecapus: an MR-compatible system for somatosensory stimulation. Neuroimage 34, 1060-1073.

Huffman, K.J., 2001. Area 3a: topographic organization and cortical connections in marmoset monkeys. Cereb. Cortex 11, 849-867.

Jang, S.H., Seo, J.P., Ahn, S.H., Lee, M.Y., 2013. Comparison of cortical activation patterns by somatosensory stimulation on the palm and dorsum of the hand. Somatosens. Mot. Res. 30, 109-113.

Johansson, R.S., Flanagan, J.R., 2009. Coding and use of tactile signals from the fingertips in object manipulation tasks. Nat. Rev. Neurosci. 10, 345-359.

Kaas, J.H., 1983a. What, if anything, is SI? Organization of first somatosensory area of cortex. Physiol. Rev. 63, 206-231.

Kaas, J.H., 1983b. What, if anything, is SI? Organization of first somatosensory area of cortex. Physiol. Rev. 63, 206-231.

Katus, T., Grubert, A., Eimer, M., 2015. Electrophysiological evidence for a sensory recruitment model of somatosensory working memory. Cereb. Cortex 25, 4697-4703.

Knight, F.L.C., Longo, M.R., Bremner, A.J., 2014. Categorical perception of tactile distance. Cognition 131, 254-262.

Kriegeskorte, N., Formisano, E., Sorger, B., Goebel, R., 2007. Individual faces elicit distinct response patterns in human anterior temporal cortex. Proc. Natl. Acad. Sci. USA 104, 20600-20605.

Kriegeskorte, N., Goebel, R., Bandettini, P.A., 2006. Information-based functional brain mapping. Proc. Natl. Acad. Sci. USA 103, 3863-3868.

Kriegeskorte, N., Mur, M., Bandettini, P.A., 2008a. Representational similarity analysis connecting the branches of systems neuroscience. Front. Syst. Neurosci. 2, 4.

Kriegeskorte, N., Mur, M., Ruff, D.A., Kiani, R., Bodurka, J., Esteky, H., Tanaka, K., Bandettini, P.A., 2008b. Matching categorical object representations in inferior temporal cortex of man and monkey. Neuron 60, 1126-1141.

LaMotte, R.H., Mountcastle, V.B., 1979. Disorders in somesthesis following lesions of parietal lobe. J. Neurophysiol. 42, 400-419.

Lloyd, D.M., Shore, D.I., Spence, C., Calvert, G.A., 2003. Multisensory representation of limb position in human premotor cortex. Nat. Neurosci. 6, 17-18.

Longo, M.R., 2017. Distorted body representations in healthy cognition. Q. J. Exp. Psychol. 70, 378-388.

Longo, M.R., 2020. Tactile distance anisotropy on the palm: a meta-analysis. Atten. Percept. Psychophys. 82, 2137-2146.

Longo, M.R., Amoruso, E., Calzolari, E., Ben Yehuda, M., Haggard, P., Azañón, E, 2020. Anisotropies of tactile distance perception on the face. Atten. Percept. Psychophys. $82,3636-3647$

Longo, M.R., Ghosh, A., Yahya, T., 2015a. Bilateral symmetry of distortions of tactile size perception. Perception 44, 1251-1262.

Longo, M.R., Golubova, O., 2017. Mapping the internal geometry of tactile space. J. Exp. Psychol. Hum. Percept. Perform. 43, 1815-1827.

Longo, M.R., Haggard, P., 2010. An implicit body representation underlying human position sense. Proc. Natl Acad. Sci. 107, 11727-11732.

Longo, M.R., Haggard, P., 2011. Weber's illusion and body shape: anisotropy of tactile size perception on the hand. J. Exp. Psychol. Hum. Percept. Perform. 37, 720-726.

Longo, M.R., Haggard, P., 2012. Implicit body representations and the conscious body image. Acta Psychol. (Amst) 141, 164-168.

Longo, M.R., Mancini, F., Haggard, P., 2015b. Implicit body representations and tactile spatial remapping. Acta Psychol. (Amst) 160, 77-87.

Longo, M.R., Morcom, R., 2016. No correlation between distorted body representations underlying tactile distance perception and position sense. Front. Hum. Neurosci. 10, 593.

Makris, N., Kennedy, D.N., McInerney, S., Sorensen, A.G., Wang, R., Caviness Jr, V.S., Pandya, D.N, 2005. Segmentation of subcomponents within the superior longitudinal fascicle in humans: a quantitative, in vivo, DT-MRI study. Cereb. Cortex 15, 854-869.

Mao, T., Kusefoglu, D., Hooks, B.M., Huber, D., Petreanu, L., Svoboda, K., 2011. Long-range neuronal circuits underlying the interaction between sensory and motor cortex. Neuron 72, 111-123.

Marks, L.E., Girvin, J.P., Quest, D.O., Antunes, J.L., Ning, P., O'Keefe, M.D., Dobelle, W.H, 1982. Electrocutaneous stimulation II. The estimation of distance between two points. Percept. Psychophys. 32, 529-536.

Matyas, F., Sreenivasan, V., Marbach, F., Wacongne, C., Barsy, B., Mateo, C., Aronoff, R., Petersen, C.C.H., 2010. Motor control by sensory cortex. Science (80-) 330, $1240-1243$.

Miller, L.E., Longo, M.R., Saygin, A.P., 2014. Tool morphology constrains the effects of tool use on body representations. J. Exp. Psychol. Hum. Percept. Perform. 40, 2143-2153.

Morgan, C., Schwarzkopf, D.S., 2020. Comparison of human population receptive field estimates between scanners and the effect of temporal filtering. F1000Res 8, 1681.

Nelson, A.J., Staines, W.R., McIlroy, W.E., 2004. Tactile stimulus predictability modulates activity in a tactile-motor cortical network. Exp. Brain Res. 154, 22-32.

Oldfield, R.C., 1971. The assessment and analysis of handedness: the Edinburgh inventory. Neuropsychologia 9, 97-113.
Oosterhof, N.N., Connolly, A.C., Haxby, J.V., 2016a. CoSMoMVPA: multi-modal multivariate pattern analysis of neuroimaging data in matlab/GNU Octave. Front. Neuroinform. $10,27$.

Oosterhof, N.N., Connolly, A.C., Haxby, J.V., 2016b. CoSMoMVPA: multi-modal multivariate pattern analysis of neuroimaging data in matlab/GNU octave. Front. Neuroinform. 10.

Oosterhof, N.N., Tipper, S.P., Downing, P.E., 2012. Viewpoint (In)dependence of action representations: an MVPA study. J. Cognit. Neurosci. 24, 975-989.

Oosterhof, N.N., Wiestler, T., Downing, P.E., Diedrichsen, J., 2011. A comparison of volume-based and surface-based multi-voxel pattern analysis. Neuroimage 56, 593-600.

Penfield, W., Boldrey, E., 1937. Somatic motor and sensory representation in the cerebral cortex of man as studied by electrical stimulation. Brain 60, 389-443.

Proklova, D., Kaiser, D., Peelen, M.V., 2016. Disentangling representations of object shape and object category in human visual cortex: the animate-inanimate distinction. J. Cognit. Neurosci. 28, 680-692.

Rohlf, F.J., Slice, D., 1990. Extensions of the procrustes method for the optimal superimposition of landmarks. Syst. Zool. 39, 40.

Rossi, S., Pasqualetti, P., Tecchio, F., Sabato, A., Rossini, P.M., 1998. Modulation of corticospinal output to human hand muscles following deprivation of sensory feedback. Neuroimage 8, 163-175.

Roux, F.-.E., Djidjeli, I., Durand, J.-.B., 2018. Functional architecture of the somatosensory homunculus detected by electrostimulation. J. Physiol. 596, 941-956.

Sakata, H., Takaoka, Y., Kawarasaki, A., Shibutani, H., 1973. Somatosensory properties of neurons in the superior parietal cortex (area 5) of the rhesus monkey. Brain Res. 64, 85-102.

Sanchez-Panchuelo, R.M., Francis, S., Bowtell, R., Schluppeck, D., 2010. Mapping human somatosensory cortex in individual subjects with 7T functional MRI. J. Neurophysiol. 103, 2544-2556.

Sereno, A.B., Lehky, S.R., 2011. Population coding of visual space: comparison of spatial representations in dorsal and ventral pathways. Front. Comput. Neurosci. 4.

Shepard, R.N., 1980. Multidimensional scaling, tree-fitting, and clustering. Science (80-) 210, 390-398.

Shinoura, N., Suzuki, Y., Yamada, R., Kodama, T., Takahashi, M., Yagi, K., 2005. Fibers connecting the primary motor and sensory areas play a role in grasp stability of the hand. Neuroimage 25, 936-941.

Sood, M.R., Sereno, M.I., 2016. Areas activated during naturalistic reading comprehension overlap topological visual, auditory, and somatotomotor maps. Hum. Brain Mapp. 37, 2784-2810.

Sperandio, I., Chouinard, P.A., Goodale, M.A., 2012. Retinotopic activity in V1 reflects the perceived and not the retinal size of an afterimage. Nat. Neurosci. 15, 540542.

Spitoni, G.F., Pireddu, G., Cimmino, R.L., Galati, G., Priori, A., Lavidor, M., Jacobson, L., Pizzamiglio, L., 2013. Right but not left angular gyrus modulates the metric component of the mental body representation: a tDCS study. Exp. Brain Res. 228, 63-72.

Stelzer, J., Chen, Y., Turner, R., 2013. Statistical inference and multiple testing correction in classification-based multi-voxel pattern analysis (MVPA): random permutations and cluster size control. Neuroimage 65, 69-82.

Stepniewska, I., Preuss, T.M., Kaas, J.H., 1993. Architectonics, somatotopic organization, and ipsilateral cortical connections of the primary motor area (M1) of owl monkeys. J. Comp. Neurol. 330, 238-271.

Stone, K.D., Keizer, A., Dijkerman, H.C., 2018. The influence of vision, touch, and proprioception on body representation of the lower limbs. Acta Psychol. (Amst) 185, 22-32.

Strick, P.L., Preston, J.B., 1982. Two representations of the hand in area 4 of a primate. II. Somatosensory input organization. J. Neurophysiol. 48, 150-159.

Sur, M., Merzenich, M.M., Kaas, J.H., 1980. Magnification, receptive-field area, and "hypercolumn" size in areas $3 \mathrm{~b}$ and 1 of somatosensory cortex in owl monkeys. J. Neurophysiol. 44, 295-311.

Tajadura Jimenez, A., Väljamäe, A., Toshima, I., Kimura, T., Tsakiris, M., Kitagawa, N, 2012. Action sounds recalibrate perceived tactile distance. Curr. Biol. 22, R516-R517.

Tamè, L., Braun, C., Holmes, N.P., Farnè, A., Pavani, F., 2016. Bilateral representations of touch in the primary somatosensory cortex. Cognit. Neuropsychol. 33, 48-66.

Tamè, L., Braun, C., Lingnau, A., Schwarzbach, J., Demarchi, G., Li Hegner, Y., Farnè, A., Pavani, F, 2012. The contribution of primary and secondary somatosensory cortices to the representation of body parts and body sides: an fMRI adaptation study. J. Cognit. Neurosci. 24, 2306-2320.

Tamè, L., Bumpus, N., Linkenauger, S.A., Longo, M.R., 2017. Distorted body representations are robust to differences in experimental instructions. Atten. Percept. Psychophys. 79 (4), 1204-1216.

Tamè, L., Holmes, N.P., 2016. Involvement of human primary somatosensory cortex in vibrotactile detection depends on task demand. Neuroimage 138, 184-196.

Tamè, L., Pavani, F., Braun, C., Salemme, R., Farnè, A., Reilly, K.T., 2015a. Somatotopy and temporal dynamics of sensorimotor interactions: evidence from double afferent inhibition. Eur. J. Neurosci. 41, 1459-1465.

Tamè, L., Pavani, F., Papadelis, C., Farnè, A., Braun, C., 2015b. Early integration of bilateral touch in the primary somatosensory cortex. Hum. Brain Mapp. 36, 1506-1523.

Taylor-Clarke, M., Jacobsen, P., Haggard, P., 2004. Keeping the world a constant size: object constancy in human touch. Nat. Neurosci. 7, 219-220.

Ten Berge, J.M.F, 1977. Orthogonal procrustes rotation for two or more matrices. Psychometrika 42, 267-276.

Tosi, G., Romano, D., 2020. The longer the reference, the shorter the legs: how response modality affects body perception. Atten. Percept. Psychophys. 82, 3737-3749.

Weber, E.H., 1996. De subtilitate tactus. In: E.H. Weber on the Tactile Senses. 2nd ed. Academic Press, London, pp. 21-128 (Original work pubilshed 1834).

Yamamoto, S., Kitazawa, S., 2001. Reversal of subjective temporal order due to arm crossing. Nat. Neurosci. 4, 759-765. 